\title{
LUT
}

University

\section{Designing for Effective Interactions with Data in the Internet of Things}

Wolff Annika, Seffah Ahmed, Kortuem Gerd, van der Linden Janet

This is a Final draft

version of a publication

published by Association for Computing Machinery

in DIS '18 Companion Proceedings of the 2018 ACM Conference Companion Publication on Designing Interactive Systems

DOI: $10.1145 / 3197391.3197402$

Copyright of the original publication: () 2019 ACM, Inc.

Please cite the publication as follows:

Annika Wolff, Ahmed Seffah, Gerd Kortuem, and Janet van der Linden. 2018. Designing for Effective Interactions with Data in the Internet of Things. In Proceedings of the 2018 ACM Conference Companion Publication on Designing Interactive Systems (DIS '18 Companion). ACM, New York, NY, USA, 415-418. DOI: https://doi.org/10.1145/3197391.3197402

This is a parallel published version of an original publication. This version can differ from the original published article. 


\section{Designing for Effective Interactions with Data in the Internet of Things (1 day workshop)}

Annika Wolff

Ahmed Seffah

Human/HCI Side of Cyber

Physical Systems

Lappeenranta University of

Technology, Finland

Annika.wolff@lut.fi

Ahmed.seffah@lut.fi

Gerd Kortuem

Design Engineering

TU Delft, The Netherlands

g.w.kortuem@tudelft.nl
Janet van der Linden

Department of Computing and

Communications

The Open University, UK

Janet.vanderlinden@open.ac.uk

\begin{abstract}
The Internet of Things (IoT), a type of cyber-physical system, has led to a drastic growth in the number of devices and sensors connected to each other and to the digital word. This has further led to an exponential increase in the amount of data being produced and disseminated throughout such systems.
\end{abstract}

This data has the potential to provide valuable insights into user behavior that can inform a design process. It also comprises an important aspect of an IoT product or service that an end user might interact to gain actionable insights. For example, when to use energy in the home, how to avoid polluted or flooded areas, or to visit the shops at quiet times. These same users may also be one source of the data that is analysed to provide this intelligence. However, in many case more intelligence is gained by combining different data sets.

This raises questions about how to help both designers and end-users to get the most value from the insights acquired through the combination and analysis of IoT data, whilst being sensitive to issues around privacy and security of data contributed by the public. There is currently no clear framework to support designers in 
navigating through a design process that uses and combines such complex data.

The aim of this one day workshop is to explore how to effectively incorporate data into a design process and how to design for more effective interactions between humans and data within IoT technologies. It will also create a roadmap for development of new methods and tools to support responsible, data-driven, co-design of new IoT interactive products and services.

\section{Author Keywords}

Human-data-interaction; Design; Internet of Things

\section{ACM Classification Keywords}

H.1.2 User/Machine Systems

\section{Related work}

Human-data interaction

Human-Data Interaction (HDI) is an emerging field of research grounded in the tradition of $\mathrm{HCI}$ and some related disciplines such media and information studies. Coined by researchers from the MIT Media Lab, in a common definition HDI refers to the collection, storage, analysis and use of personal data (Mortier et al., 2014). However, in the context of the new emerging cyberphysical and IoT technologies connecting digital artifacts and physical objects world there is a broader definition, providing a larger range of potential applications and challenges of HDI. In this definition, a wider range of data sources should be considered, including: data that is private and personal; data that is open; data deriving from the environment from networked sensors; data both from and about people. These datasets, when put together or linked by and via humans, have the same importance and consequences as the personal data in the original HDI definition. In this workshop, HDI is also about the interactive technologies and interaction patterns needed to achieve the following tasks (Seffah, 2017):

- Engage users with data

- Connect users with data and connect datasets to each other

- Collect data

- Extract meaning from data to answer specific questions

Data-driven design of data-intensive IoT services One technological context in which the study of HDI is particularly important is to support the responsible design of new Internet of Things (IoT) technologies, which interconnects physical devices, via the internet, to each other and to people and which utilize large quantities of very diverse data. IoT technologies may comprise varying combinations of networked sensors, actuators, complex datasets, algorithms and human interfaces - often collecting data from humans and providing insights back to them.

Recent research related to the use and role of data within IoT have investigated the perceived usefulness of data the ability of people - especially non-professionals - to design using data and the types of mental models that people have when interacting with IoT technologies. The Datacatcher (Gaver et al., 2016) explores how people would interact with data that was relevant to a person's current geographical location and presented through a bespoke device. The Physikit (Houben et al, 2016) explored how people could conceive and programme their own IoT devices using a kit of sensors and actuators and a simple programming environment. In the same 
vein Bitbarista (Pschetz et al., 2017) also explored how to make IoT data more salient in an effort to increase engagement and positive feelings towards it. Yarosh and Zave (2017) identified errors that people might make when interacting with IoT technologies, due to faulty mental models.

IoT technologies may also be used to collect data as part of a data-driven design process. The benefit is that they are able to collect data at a scale that can be analyzed to provide insight into patterns of use and behavior. Examples might include data collected from mobile phones, or data collected from sensors, which might be attached directly to people through other wearable devices, or embedded into products, or into the environment itself.

\section{Challenges}

Whether data is being collected to inform design, to make decisions, to comprehend a phenomena or whether it is the essence of the final designed service, if the data is either from or about people this raises concerns over privacy and use of such data. Where the data is being analyzed and presented to people, this raises questions as to how users will interact with data in what form it should be presented and through what sort of media and devices.

The above identifies a number of design challenges that require:

- Broadening the vision of interactions beyond the traditional human-computer interaction, to include interactions with other physical devices and interfaces to these devices. Examples could include wearables, public displays, interactivity embedded into physical space, such as smart lighting, or even smart walls, to name a few.
- Connecting different methods for designing data-intensive smart, interactive services and user interfaces

- $\quad$ Building a tradeoff between the attributes that quantify the user experiences and those that measure security and privacy. Most often these factors are seen as obstacles in interaction design

The benefit and challenges of co-design

A co-design process, which gives end-users a more active role in the design process, may help to both better understand user requirements and to mitigate against concerns about data use and privacy. The benefits of a co-design approach are well established and include better knowledge of end-user needs, improved creativity and higher customer satisfaction (Steen, Manschot \& De Koning, 2011).

While there are many co-design methods and tools that support a general design process, there is a growing need to provide better support both for designers and end-users to work with and make sense of complex data during the design of new IoT technologies. While there is currently research into different areas of HDI, it is fragmented and there is no clear idea as to how differing perspectives might serve to support co-design around complex data.

The workshop aims to provide better clarity regarding the diverse ways in which humans and data are interacting, both in the process of collecting and analyzing data that informs a design and when it is integrated into the functioning of an end-product. Through this process, a roadmap for this research field will be produced.

The core areas of interest are: 
- HCI design theories and methods and how they apply to Human-data interaction within IoT

- Interaction with networked physical objects with embedded sensors

- Innovative approaches to smart city dataintensive services design in the context of IoT

- User research and empirical studies on HDI within IoT

- Novel interaction technologies and models for IoT

- Interactions and visualization of data in the physical space (e.g. data physicality)

- Building data literacy of stakeholders through a design process to support better co-creation around data

- Ethical, privacy and usability concerns around interactions between humans and IoT technologies

The ACM DIS conference and research community is an ideal forum for bringing a multidisciplinary perspective to understanding the design challenges with complex data around environments, people and connected objects and for understanding how to design for better interactions between humans and the data used in the design of smart services.

This workshop aims to be the starting point for bringing together designers, technologists, data experts and $\mathrm{HCI}$ practitioners who have an interest and expertise in this emerging field. The goal is to help to define the field "HDI for Internet of Things", identify the different technological trends and build a research roadmap to take the field forward into the future.

\section{References}

1. William Gaver, Andy Boucher, Nadine Jarvis, David Cameron, Mark Hauenstein, Sarah Pennington, John Bowers, James Pike, Robin Beitra, and Liliana Ovalle.
2016. The Datacatcher: Batch Deployment and Documentation of 130 Location-Aware, Mobile Devices That Put Sociopolitically-Relevant Big Data in People's Hands: Polyphonic Interpretation at Scale. In Proceedings of the 2016 CHI Conference on Human Factors in Computing Systems (CHI '16). ACM, New York, NY, USA, 1597-1607. DOI: https://doi.org/10.1145/2858036.2858472

2. Steven Houben, Connie Golsteijn, Sarah Gallacher, Rose Johnson, Saskia Bakker, Nicolai Marquardt, Licia Capra, and Yvonne Rogers. 2016. Physikit: Data Engagement Through Physical Ambient Visualizations in the Home. In Proceedings of the 2016 CHI Conference on Human Factors in Computing Systems (CHI '16). ACM, New York, NY, Computing Systems (CHI '16). ACM, New York, NY,
USA, 1608-1619. DOI:

https://doi.org/10.1145/2858036.2858059

3. Larissa Pschetz, Ella Tallyn, Rory Gianni, and Chris Speed. 2017. Bitbarista: Exploring Perceptions of Data Transactions in the Internet of Things. In Proceedings of the 2017 CHI Conference on Human Factors in Computing Systems (CHI '17). ACM, New York, NY, USA, 2964-2975. DOI: https://doi.org/10.1145/3025453.3025878

4. Mortier, R., Haddadi, H., Henderson, T., McAuley, D. and Crowcroft, J., 2014. Human-data interaction: The human face of the data-driven society.

5. Steen, M., Manschot, M. A. J., \& De Koning, N. (2011). Benefits of co-design in service design projects. International Journal of Design 5 (2) 2011, 53-60.

6. Yarosh, L., \& Zave, P. (2017, May). Locked or Not?: Mental Models of IoT Feature Interaction. In Proceedings of the $2017 \mathrm{CHI}$ Conference on Human Factors in Computing Systems (pp. 2993-2997). ACM. 